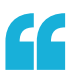

these results

... are

promising and

support the

further testing

of C. novyi-NT

for treatment

of inoperable

therapy-

resistant

tumours

in humans

\title{
THERAPEUTICS
}

\section{Bacterial treatment for cancer}

A new study by Roberts and colleagues has shown that intratumoural injection of an attenuated version of Clostridium novyi bacteria can reduce tumour volume in several preclinical animal models as well as in one human.

C. novyi can induce tumour cell lysis, is very sensitive to oxygen levels and thrives in hypoxic environments, such as those often found in advanced solid tumours, but does not grow in normoxic environments, such as those typically found in normal tissue. Roberts et al. hypothesized that these characteristics would make it an ideal therapy for tumours that cannot be removed surgically, such as gliomas.

The authors had previously treated tumours in mice and rabbits intravenously with an attenuated C. novyi strain (C. novyi-NT) and observed promising response rates but could not recapitulate these responses in larger canine models. As this might be due to inadequate delivery to the tumour, the authors tested intratumoural injections of C. novyi-NT, initially in a rat model of highly aggressive gliomas. Treatment of these rat brain tumours with intratumoural injections caused a significant decrease in tumour burden within 48 hours of the injection and spared surrounding normal cells that were only micrometres away. Although brain oedemas were commonly seen in these animals, this adverse effect was easily managed.

The authors next tested this treatment in dogs, as dogs often develop spontaneous solid tumours and thus might more accurately model human tumours. Intratumoural injection of C. novyi-NT spores into a variety of solid tumours in dogs resulted in an objective response in $37.5 \%$ of all dogs $(n=16)$. Half of the dogs that showed objective responses had long-term responses. Adverse events were related to bacterial infection and could be controlled with antibiotics.

Owing to the success in the canine models, the authors set up a phase I investigational clinical trial and enrolled a patient with a late-stage metastatic retroperitoneal leiomyosarcoma. One of the metastases, located in the patient's shoulder, was targeted for treatment with C. novyiNT spores. The treatment reduced the tumour volume although the patient also developed adverse effects related to bacterial infection, such as fever, which was managed with antibiotics.
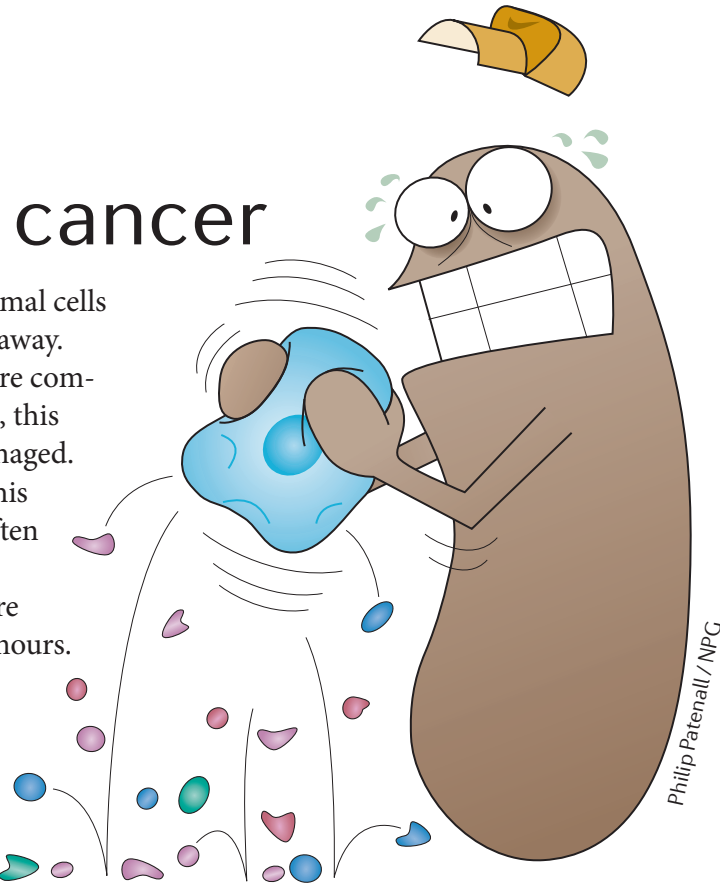

The clinical trial is ongoing, and the authors outline several avenues of further research including determining the role of acquired immunity in the response to C. novyi-NT treatment, and whether this therapy could be combined with established chemotherapies and radiotherapies. Thus, although these results are preliminary, they are promising and support the further testing of C. novyiNT for treatment of inoperable therapy-resistant tumours in humans. Isabel Lokody

ORIGINAL RESEARCH PAPER Roberts, N. J. et al. Intratumoral injection of Clostridium novyi-NT spores induces antitumor responses. Sci. Trans Med. 6, 249ra111 (2014) 